\title{
Critical evaluation of the colossal Seebeck coefficient of nanostructured rutile $\mathrm{MnO}_{2}$
}

Denis Music and Jochen M. Schneider

Materials Chemistry, RWTH Aachen University, Kopernikusstr. 10, 52074 Aachen, Germany

E-mail: music@mch.rwth-aachen.de

\begin{abstract}
We have explored the correlation between the Seebeck coefficient and the electronic structure of nanostructured rutile $\mathrm{MnO}_{2}$ using density functional theory to critically appraise the three orders of magnitude scatter in literature data. Our hypothesis is that the microstructure and morphology on the nanoscale is causing this behaviour, which we have tested by comparing the Seebeck coefficient of bulk $\mathrm{MnO}_{2}$ with two low-energy surfaces: $\mathrm{MnO}_{2}(110)$ and $\mathrm{MnO}_{2}(001)$. From these data, it is evident that variations over two orders of magnitude in the Seebeck coefficient can be attained by affecting domain size and texture on the nanoscale. This may be understood by analysing the electronic structure. Surface hybridized $\mathrm{Mn} d-\mathrm{O} p$ states fill the band gap of $\mathrm{MnO}_{2}$ and thus substantially alter the transport properties.
\end{abstract}

Keywords: $\mathrm{MnO}_{2}$, Seebeck coefficient, density functional theory

(Some figures may appear in colour only in the online journal) 


\section{Introduction}

Thermoelectrics are a possible solution of the emerging global need for renewable energy sources with zero greenhouse-gas emission [1]. Extensive scientific efforts have been dedicated towards increasing a figure of merit, a measure of their efficiency, which involves maximizing the Seebeck coefficient and electrical conductivity as well as minimizing the thermal conductivity of these devices [1]. As these transport characteristics depend on several, in a complex fashion, interrelated physical properties, efficiency enhancement is challenging [1]. For instance, charge carriers give rise to both electrical and thermal conductivities, where the thermal conductivity also exhibits phonon contributions [1]. To disrupt the phonon paths, alloying with large elements or nanostructuring may be attempted [1]. It should also be mentioned that the electronic structure is substantially affected by lowering the dimensionality of a crystal via nanostructuring as quantum confined states can appear close to the Fermi level and hence drastically increase the Seebeck coefficient [2-4]. For instance, InAs nanowires, i.e. 1D structures, were reported to exhibit an order of magnitude increased efficiency with respect to bulk [5]. An even larger enhancement of the efficiency may be achieved in nanostructured hierarchical structures [6].

Another very promising approach to enhance the performance of thermoelectric devices is to design phases with enormous Seebeck coefficients, which are sometimes referred to as colossal or giant Seebeck coefficients. For instance, at $10 \mathrm{~K}$ the Seebeck coefficient of $60000 \mu \mathrm{V} \mathrm{K}^{-1}$ was reported for oxygen deficient rutile $\mathrm{TiO}_{2}$ single crystals [7]. For comparison, commercial $n$-doped $\mathrm{Bi}_{2} \mathrm{Te}_{3}$ exhibits the Seebeck coefficient of $-200 \mu \mathrm{V} \mathrm{K}{ }^{-1}$ at $300 \mathrm{~K}$ [8]. Another well investigated system is strongly correlated $\mathrm{FeSb}_{2}$ with the Seebeck coefficient of $-45000 \mu \mathrm{V} \mathrm{K}^{-1}$ at $10 \mathrm{~K}$ [9]. It is typical for these colossal Seebeck coefficient systems to exhibit a peak performance at very low temperatures [7, 9-11], which limits their possible applications to refrigeration or sensors, as power generation is not viable since large 
temperature gradients are required to obtain enhanced Carnot efficiencies. A system that appears promising at higher temperatures is $\mathrm{MnO}_{2}$ (space group $P 4_{2} / m n m$, prototype rutile). No other $\mathrm{MnO}_{2}$ polymorphs have been discussed in terms of the Seebeck coefficient measurements. A very large spread of the Seebeck coefficient has been reported, ranging from -80 to $-20000 \mu \mathrm{V} \mathrm{K}^{-1}$ at $300 \mathrm{~K}$ [12-15]. However, the physical reason for this spread over three orders of magnitude is unknown.

In this work, we seek out the correlation of the Seebeck coefficient and the electronic structure of nanostructured $\mathrm{MnO}_{2}$ using density functional theory to critically appraise the large scatter in of the reported values [12-15]. A working hypothesis is that the microstructure and morphology on the nanoscale is causing such behaviour. However, when thermoelectrics are explored using density functional theory, these low-dimensionality aspects are very commonly disregarded due to more demanding computational requirements. We calculate the Seebeck coefficient of bulk $\mathrm{MnO}_{2}$ and two low-energy surfaces. We show that by controlling the domain size and texture of nanostructured $\mathrm{MnO}_{2}$, two orders of magnitude modulation of the Seebeck coefficient can be expected.

\section{Theoretical methods}

Density functional theory [16] was used within the framework of the Vienna $a b$ initio simulation package (VASP) with projector augmented wave potentials [17-19]. These electronic potentials were parameterized within the generalized-gradient approximation by Perdew, Burke and Ernzerhof [20]. The Blöchl corrections were employed for the total energy [21] and the integration in the Brillouin zone was made on Monkhorst-Pack $k$-points [22]. Rutile $2 \times 2 \times 2$ supercells containing 48 atoms were studied on a mesh of $6 \times 6 \times 6 \mathrm{k}$-points. To account for strong correlations, the so-called Hubbard approach was used with the Coulomb and exchange parameters of 6.7 and $1.2 \mathrm{eV}$, respectively [23]. Antiferromagnetic ground state 
was considered [23]. Full structural optimization was carried out for each configuration. The convergence criterion for the total energy was $0.01 \mathrm{meV}$ within a $500 \mathrm{eV}$ cut-off. Ability of using ground state theories such as density functionally theory to calculate thermoelectric properties was confirmed previously [24-28]. Here, the Seebeck coefficient $(S)$ was estimated from the density of states $(D)$ at the gamma point using the semiclassical Boltzmann transport theory under the assumption of constant relaxation time:

$$
S=\frac{k}{q} \frac{\int_{-\infty}^{+\infty} \frac{f}{(f+1)^{2}} D(E) \frac{E-E_{F}}{k T} d E}{\int_{-\infty}^{+\infty} \frac{f}{(f+1)^{2}} D(E) d E}
$$

where $k, q, E, E_{F}$ and $T$ designate the Boltzmann constant, unit charge, total energy, Fermi energy and absolute temperature, respectively [27]. The term $f$ stands for $\exp \left(\left(E-E_{F}\right) / k T\right)$. Here, $T$ of $300 \mathrm{~K}$ was considered. Using equation (1) to obtain the Seebeck coefficient yields deviations up to $30 \%$ between measured and calculated values as many approximations are made, such as resonance scattering, phonon drag, anisotropy and size effects are ignored as well as constant relaxation time is assumed [3]. Nevertheless, considering ideal solids with band structure centred at the highest symmetry point (gamma point) still provides useful trends for experiments [3, 24-29].

\section{Results and discussion}

As two low-energy surfaces of $\mathrm{MnO}_{2}$ are $\mathrm{O}$ terminated (110) and $\mathrm{Mn}-\mathrm{O}$ terminated (001), with the (110) surface being the lowest energy configuration [30], we test our working hypothesis that the microstructure and morphology on the nanoscale cause large variations of the Seebeck coefficient by comparing the electronic structure of bulk and surface $\mathrm{MnO}_{2}$. Figure 1 shows the electron density distributions plots for bulk $\mathrm{MnO}_{2}$ as well as $\mathrm{MnO}_{2}(110)$ and $\mathrm{MnO}_{2}(001)$. Size effects are not expected here as the critical surface slab thickness is 
reached [31]. $\mathrm{Mn}$ and $\mathrm{O}$ atoms are hybridized in the bulk state, as indicated by the shared electron distribution, and there is a substantial charge difference between these two constituents, pointing out at a mixture of covalent and ionic bonding. As the surfaces are formed, considerable changes in the electronic structure can be observed. The (001) surface is characterized by bond breaking and as a result thereof a very strong in-plane bonding between $\mathrm{Mn}$ and $\mathrm{O}$ atoms is present. On the other hand, the (110) surface exhibits outward buckling of surface $\mathrm{O}$ atoms, which is likely a compensation for bond breaking upon surface formation. These surface $\mathrm{O}$ atoms are less strongly bonded to $\mathrm{Mn}$ atoms in the underlying layer. Similar relaxations effects are observed for the (001) surface as well. These observations are consistent with literature [30]. Clearly, there is a difference in the chemical bonding between bulk and both of these surfaces, which may in turn affect the transport properties. 

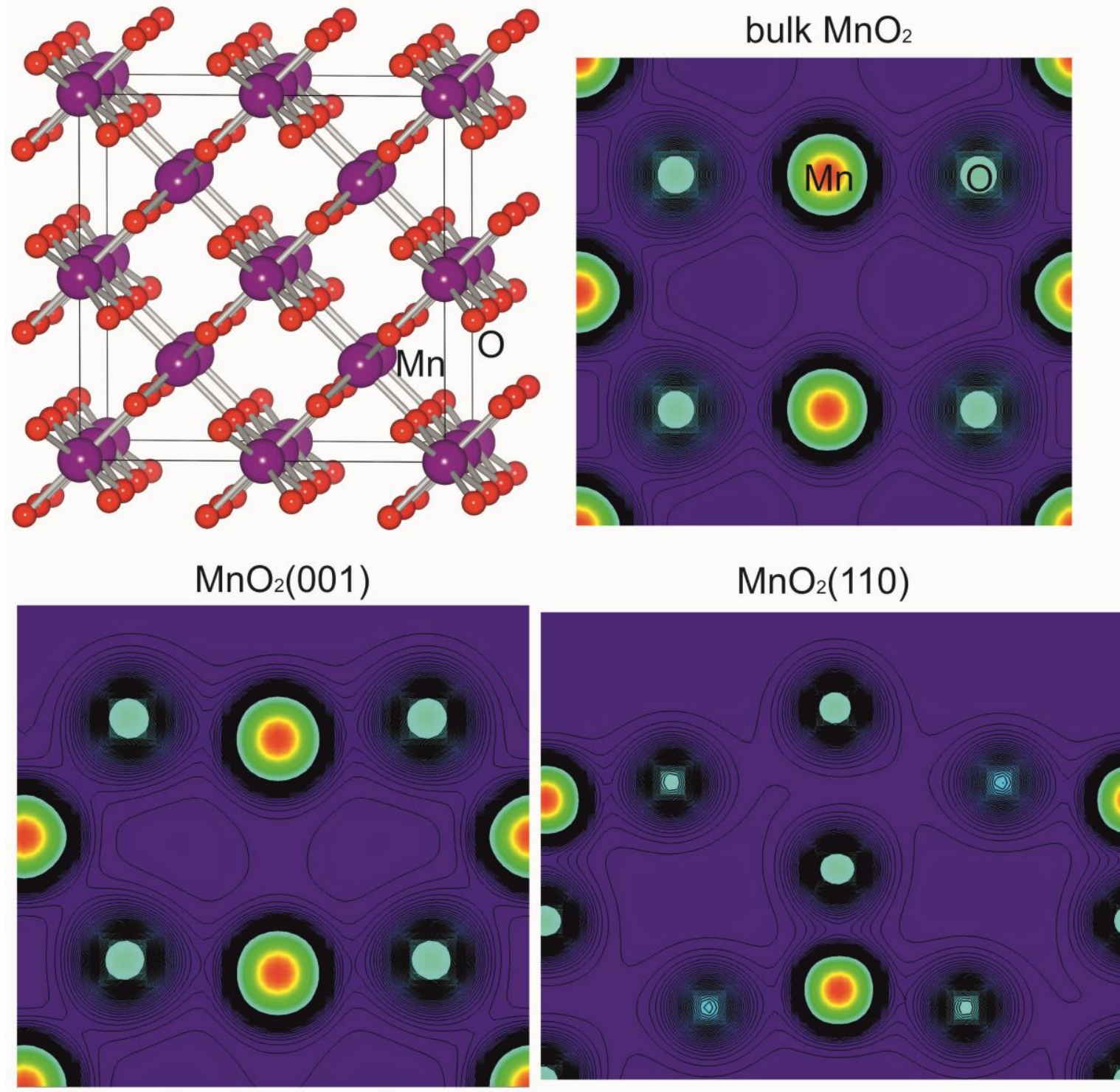

\section{$0.0-3.9$ electrons $/ \AA^{3}$}

Figure 1. Crystal structure of $\mathrm{MnO}_{2}$ together with vertical cut through the electron density distributions of bulk $\mathrm{MnO}_{2}$ as well as $\mathrm{MnO}_{2}(001)$ and $\mathrm{MnO}_{2}(110)$.

A more detailed analysis of the electronic structure of bulk $\mathrm{MnO}_{2}, \mathrm{MnO}_{2}(110)$ and $\mathrm{MnO}_{2}(001)$ is carried out by evaluating the density of states. The corresponding density of states data are provided in figure 2. It is obvious that bulk $\mathrm{MnO}_{2}$ is a semiconductor as a band gap is opened at the Fermi level, which is consistent with literature [23, 12]. By forming (110) and (001) surfaces, the band gap of bulk $\mathrm{MnO}_{2}$ is partly filled with surface states for 
$\mathrm{MnO}_{2}(110)$ and completely filled for $\mathrm{MnO}_{2}(001)$. As the (110) surface reconstructs by outward buckling of $\mathrm{O}$ atoms, less surface states are allocated in the band gap for this configuration. On the other hand, the (001) surface is energetically less favoured as reconstruction does not occur so that more states fill the band gap. From the analysis of these total densities of states, it is clear that bulk $\mathrm{MnO}_{2}$ and surfaces of $\mathrm{MnO}_{2}$ exhibit subtle differences in the electronic structure as surface states fill the band gap of $\mathrm{MnO}_{2}$. To provide further insights of the physical nature of these surface states filling the band gap, we analyse the partial density of states for $\mathrm{MnO}_{2}(110)$, as a representative configuration, which is given in figure 3. In the whole energy range $\mathrm{Mn} d$ and $\mathrm{O} p$ states are hybridized. Additional surface states located in the band gap are of the same type. Hence, filling the band gap is purely a surface effect as no additional boding type is found.

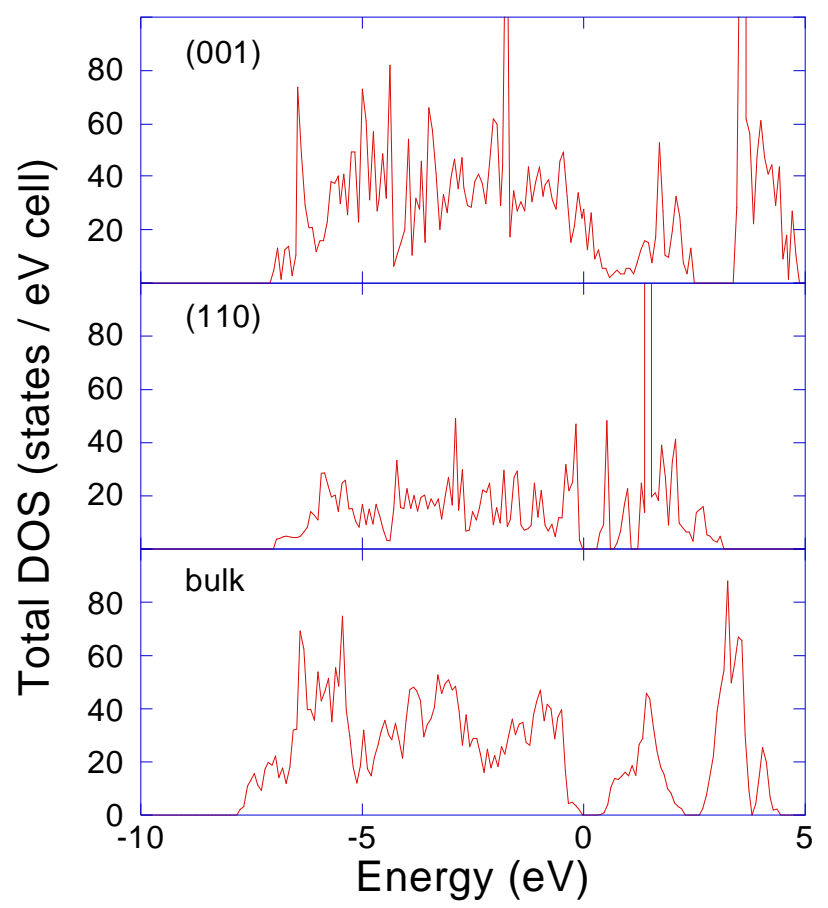

Figure 2. Total density of states (DOS) for $\mathrm{MnO}_{2}$ in the bulk form as well as its (110) and (001) surfaces. For simplicity spin up and spin down states are not distinguished. The Fermi energy is set to $0 \mathrm{eV}$. 


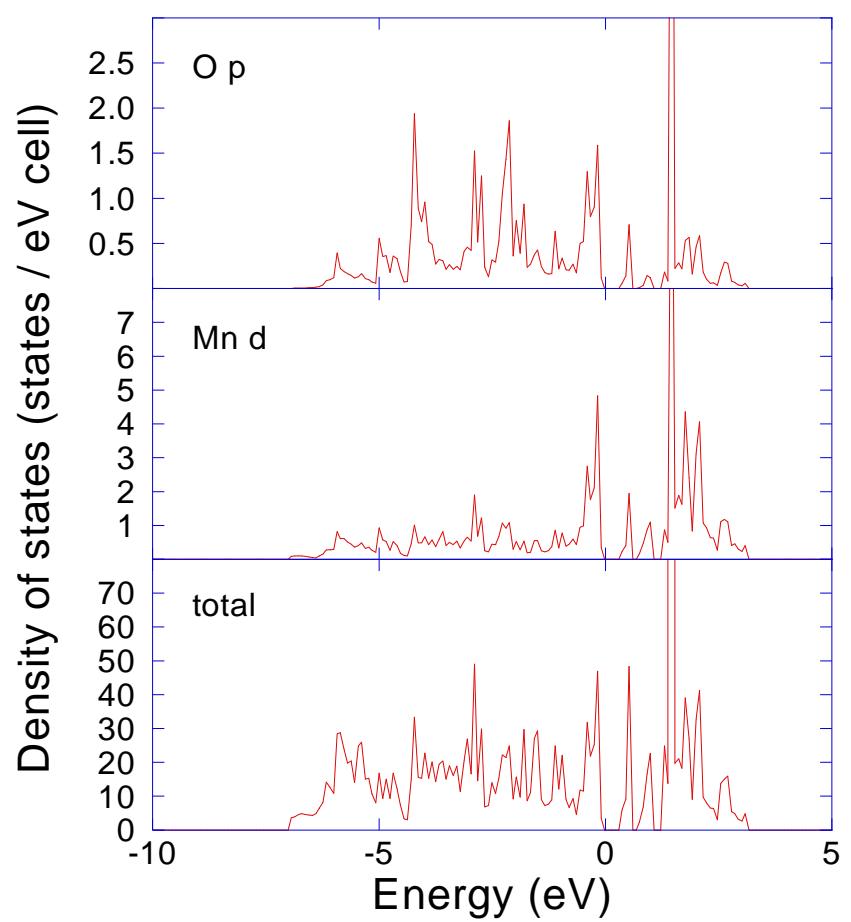

Figure 3. Total and partial density of states for $\mathrm{MnO}_{2}$ (110). For simplicity spin up and spin down states are not distinguished. The Fermi energy is set to 0 $\mathrm{eV}$.

Using the density of states data presented above (see figure 2) and equation (1), the Seebeck coefficient can be calculated. The Seebeck coefficients of bulk $\mathrm{MnO}_{2}, \mathrm{MnO}_{2}(110)$ and $\mathrm{MnO}_{2}(001)$ are $-354,-438$ and $+4 \mu \mathrm{V} \mathrm{K}^{-1}$, respectively. These data, together with the literature values, can be found in figure 4 . As there is a large scatter of the reported data in the range from -80 to $-20000 \mu \mathrm{V} \mathrm{K}^{-1}$ [12-15], we first need to critically examine the literature more closely before comparing our data to the experimental values. It is known that $\mathrm{MnO}_{2}$ possesses a large capacitance at large currents [32]. Hence, measurements performed with a DC Seebeck probe may yield ambiguous results. Sato et al. [12] as well as Walia et al. [14] used an AC probe and obtained the Seebeck coefficient of -80 and $-460 \mu \mathrm{V} \mathrm{K}^{-1}$, respectively, while Song et al [15] utilised a DC probe measuring -20 $000 \mu \mathrm{V} \mathrm{K}^{-1}$. Islam et al. [13] reported a Seebeck coefficient of $-300 \mu \mathrm{V} \mathrm{K} \mathrm{K}^{-1}$, but it is not clear whether a DC or an AC probe was 
employed. Our hypothesis is that the microstructure and morphology on the nanoscale is causing large variations of the Seebeck coefficient. Hence, the spread of our Seebeck coefficient data over two orders of magnitude is consistent with the reports of Sato et al. [12], Islam et al. [13] and Walia et al. [14]. To critically evaluate our Seebeck coefficient data, we perform more computational tests. By calculating the energy of formation of oxygen surface vacancy for $\mathrm{MnO}_{2}(110)$, we test the adequacy of our slab geometry in terms of slab thickness (up to 6 layers used here, frozen bottom layer) and vacuum thickness (15 A used here). The energy of formation of $1.1 \mathrm{eV}$ per oxygen surface vacancy is obtained, which is consistent with literature [30], where surface models were probed in terms of the slab and vacuum thickness convergence. Furthermore, we also include dipole corrections into the calculations of the Seebeck coefficient. The largest deviation of $31 \%$ occurs with respect to the surface models without dipole corrections, which does not affect the general trends. Hence, our surface models appear adequate to calculate the Seebeck coefficients of $\mathrm{MnO}_{2}$ surfaces. Furthermore, adopting an alternative Hubbard scheme with the effective Coulomb repulsion parameter of $5.1 \mathrm{eV}$ [30] results in a $6 \%$ decrease in the Seebeck coefficient. Thus, both computational strategy and surface models are acceptable. The large difference in the Seebeck coefficient for different $\mathrm{MnO}_{2}$ surfaces may be explained through the electronic structure data (see figure 3 and figure 4). Surface hybridized $\mathrm{Mn} d-\mathrm{O} p$ states fill the band gap of $\mathrm{MnO}_{2}$ and thus alter the transport properties. Grains on the nanoscale with the $\langle 110\rangle$ texture and thus large surface/volume ratios may exhibit negative and large absolute Seebeck coefficient values. Hence, it is reasonable to assume that the reported differences in measured Seebeck coefficients by Sato et al. [12], Islam et al. [13] and Walia et al. [14] are a consequence of microstructural and morphological variations. Only the paper by Walia et al. [14] contains some constitutional information. The grains are in the range of $400 \mathrm{~nm}$ and based on the diffraction data the samples appear to exhibit fully random crystallographic orientations [14]. A decrease in the grain size down to the nanoscale and a larger population of $\langle 110\rangle$ oriented 
grains may further increase the absolute value of the Seebeck coefficient, but it is unreasonable to expect colossal values. However, based on our notion that the microstructure and morphology on the nanoscale give rise to a two orders of magnitude variation in Seebeck coefficient, further increase in the absolute Seebeck coefficient values can be expected. For example, for quantum dot superlattice thermoelectric devices further increases may be possible [33], but this has not been explored for $\mathrm{MnO}_{2}$. Furthermore, 2D (multilayers or superlattices), 1D (nanowires), or 0D samples (quantum dots) may also be hierarchies to enhance the thermoelectric performance.

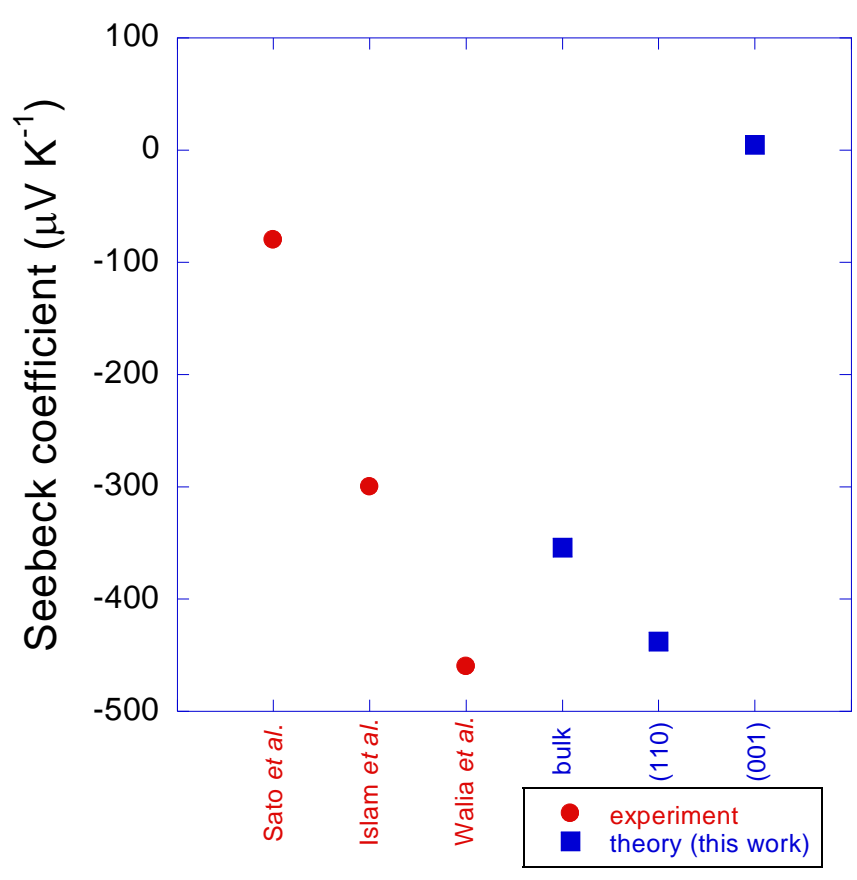

Figure 4. Experimental and calculated Seebeck coefficients for $\mathrm{MnO}_{2}$.

\section{Conclusion}

In conclusion, we have explored the correlation between the Seebeck coefficient and the electronic structure of nanostructured $\mathrm{MnO}_{2}$ to critically assess the three orders of magnitude scatter in the literature data, from -80 to $-20000 \mu \mathrm{V} \mathrm{K}^{-1}$ at $300 \mathrm{~K}$ [12-15]. Our starting hypothesis was that the microstructure and morphology on the nanoscale was causing this behaviour. To test this hypothesis we have compared the Seebeck coefficient of bulk $\mathrm{MnO}_{2}$ 
with two low-energy surfaces: $\mathrm{MnO}_{2}(110)$ and $\mathrm{MnO}_{2}(001)$. The Seebeck coefficients of bulk $\mathrm{MnO}_{2}, \mathrm{MnO}_{2}(110)$ and $\mathrm{MnO}_{2}(001)$ are $-354,-438$ and $+4 \mu \mathrm{V} \mathrm{K}^{-1}$, respectively. This spread of our Seebeck coefficient data over two orders of magnitude is consistent with the reports of Sato et al. [12], Islam et al. [13] and Walia et al. [14] This large difference in the Seebeck coefficient for different $\mathrm{MnO}_{2}$ surfaces may be understood by analysing the electronic structure. Surface hybridized $\mathrm{Mn} d-\mathrm{O} p$ states fill the band gap of $\mathrm{MnO}_{2}$ and thus substantially alter the transport properties. Colossal Seebeck coefficient of $\mathrm{MnO}_{2}$ cannot be expected for $\mathrm{MnO}_{2}$. However, a two orders of magnitude tunability of $\mathrm{MnO}_{2}$ by affecting microstructure and morphology on the nanoscale may enable applications at elevated temperatures.

\section{Acknowledgements}

This work was supported by the Deutsche Forschungsgemeinschaft (DFG) within the project SCHN 735/24-1.

\section{References}

[1] Snyder G J and Toberer E S 2008 Nature Mater. 7105

[2] Arakawa Y and Sakaki H 1982 Appl. Phys. Lett. 40939

[3] Heremans J P, Wiendlocha B and Chamoire A M 2012 Energy Environ. Sci. 55510

[4] Jood P, Mehta R J, Zhang Y, Peleckis G, Wang X, Siegel R W, Borca-Tasciuc T, Dou S X and Ramanath G 2011 Nano Lett. 114337

[5] Wu P M, Gooth J, Zianni X, Svensson S F, Gluschke J G, Dick K A, Thelander C, Nielsch K and Linke H 2013 Nano Lett. 134080

[6] Mi J-L, Lock N, Sun T, Christensen M, Søndergaard M, Hald P, Hng H H, Ma J and Iversen B B 2010 ASC Nano 42523

[7] Tang J, Wang W, Zhao G-L and Li Q 2009 J. Phys.: Condens. Matter 21205703

[8] Goldsmid A J, Sheard A R and Wright D A 1958 Br. J. Appl. Phys. 9365

[9] Bentien A, Johnsen S, Madsen G K H, Iversen B B and Steglich F 2007 EPL 8017008

[10] Sagar S, Ganesan V, Joy P A, Thomas S, Liebig A, Albrecht M and Anantharaman M R 2010 EPL 9117008

[11] Jaworski C M, Myers R C, Johnston-Halperin E and Heremans J P 2012 Nature 487 210

[12] Sato H, Enoki T, Isobe M and Ueda Y 2000 Phys. Rev. B 613563

[13] Islam A K M F U, Islam R and Khan K A 2005 J. Mater. Sci.: Mater. Electr. 16203

[14] Walia S, Balendhran S, Yi P, Yao D, Zhuiykov S, Weber M P R, Strano M S, Bhaskaran M, Sriram S and Kalantar-zadeh K 2013 J. Phys. Chem. C 1179137 
[15] Song F F, Wu L and Liang S 2012 Nanotechnol. 23085401

[16] Hohenberg P and Kohn W 1964 Phys. Rev. 136 B864

[17] Kresse G and Hafner J 1993 Phys. Rev. B 4813115

[18] Kresse G and Hafner J 1994 Phys. Rev. B 4914251

[19] Kresse G and Joubert D 1999 Phys. Rev. B 591758

[20] Perdew J P, Burke K and Ernzerhof M 1996 Phys. Rev. Lett. 773865

[21] Blöchl P E 1994 Phys. Rev. B 5017953

[22] Monkhorst H J and Pack J D 1976 Phys. Rev. B 135188

[23] Tompsett D A, Middlemiss D S and Islam M S 2012 Phys. Rev. B 86205126

[24] Seetawan T, Vora-Ud A, Chainaronk P, Thanachayanont C and Amornkitbamrung V 2010 Comput. Mater. Sci. 49 S225

[25] Music D, Basse F H-U and Schneider J M 2010 Cryst. Growth Des. 104531

[26] Ong K P, Singh D J and Wu P 2011 Phys. Rev. B 83115110

[27] Luo X, Sullivan M B and Quek S Y 2012 Phys. Rev. B 86184111

[28] Music D, Basse F H-U, Han L, Devender, Borca-Tasciuc T, Gengler J J, Voevodin A A, Ramanath G and Schneider J M 2014 Appl. Phys. Lett. 104053903

[29] Music D, Chen Y-T, Geyer R W, Bliem P and Schneider J M 2014 Mater. Res. Express 1045034

[30] Tompsett D A, Parker S C and Islam M S 2014 J. Am. Chem. Soc. 1361418

[31] Sigumonrong D P, Music D and Schneider J M 2011 Comput. Mater. Sci. 501197

[32] Xie X, Zhang C, Wu M-B, Tao Y, Lv W and Yang Q-H 2013 Chem. Comm. 4911092

[33] Harman T C, Walsh P J T M P and LaForge B E 2002 Science 2972229 